\title{
Sociologie du changement dans les entreprises et les organisations
}

De Philippe Bernoux - Paris, Editions du Seuil, 2004, 382 p.

Benoît Cordelier

\section{OpenEdition}

\section{Journals}

Édition électronique

URL : http://journals.openedition.org/communicationorganisation/3502

DOI : 10.4000/communicationorganisation.3502

ISSN : 1775-3546

Éditeur

Presses universitaires de Bordeaux

Édition imprimée

Date de publication : 1 octobre 2006

ISSN : 1168-5549

\section{Référence électronique}

Benoît Cordelier, "Sociologie du changement dans les entreprises et les organisations ",

Communication et organisation [En ligne], 30 | 2006, mis en ligne le 21 juin 2012, consulté le 21

septembre 2020. URL : http://journals.openedition.org/communicationorganisation/3502 ; DOI :

https://doi.org/10.4000/communicationorganisation.3502

Ce document a été généré automatiquement le 21 septembre 2020

(C) Presses universitaires de Bordeaux 


\section{Sociologie du changement dans les entreprises et les organisations}

De Philippe Bernoux - Paris, Editions du Seuil, 2004, 382 p.

\section{Benoît Cordelier}

1 Le travail de Philippe Bernoux est hautement pédagogique. Nous y avons été habitué dans d'autres de ses ouvrages et, ce livre ne faillit pas dans ce domaine. L'auteur nous propose en effet tout d'abord une analyse critique de divers courants de la sociologie des organisations ayant étudié le changement organisationnel, puis se penche sur les déterminants $\mathrm{du}$ changement (contraintes socio-économiques et techniques, institutions, acteurs), avant de conclure sur des pratiques de conduite du changement.

2 Philippe Bernoux veut remettre au centre les individus dans la compréhension des phénomènes de changement organisationnel en contextualisant leur action. «L'idée est qu'on ne peut raisonner sur les comportements des individus ni seulement en terme d'intérêts individuels, ni seulement en termes de contraintes par les structures, mais qu'il faut prendre en compte les relations sociales concrètes, et le contexte social dans lesquels les individus sont impliqués. » (p. 26) Ce ne sont ni les facteurs socio-économiques ou encore techniques qui peuvent expliquer le changement. Philippe Bernoux en revient vers les théories de l'action. Expliquer les transformations de l'organisation n'est pour lui possible qu'en passant par la compréhension des comportements individuels. Il favorise à cet effet une théorie de l'action interactionniste contre toute résurgence néo-taylorienne ou retour vers un réalisme totalitaire. Pour lui, toute modélisation de l'organisation et du changement organisationnel ne peut que prendre en compte l'acteur au risque de tomber dans un déterminisme technologique ou fonctionnel. La rationalité de l'acteur et le sens qu'il donne à son agir sont donc centraux.

3 Il se range donc à une approche héritée de l'individualisme méthodologique de Boudon. En effet Boudon a voulu instituer une praxéologie dans laquelle tout phénomène social est le résultat d'actions, d'attitudes, de croyances et de comportements individuels. Le sociologue, ou le chercheur en général, qui veut en analyser le fonctionnement doit alors recourir à l'étude du sens de l'action par le Verstehen wéberien. Bernoux complète cette approche par la théorie de l'encastrement (embeddedness) qui tient à la prise en 
compte des relations sociales concrètes et du contexte dans lequel elles se déroulent. Ici, le changement ne peut avoir lieu qu'à partir du moment ou l'acteur lui donne un sens et que l'institution en garantisse le respect : pour Bernoux, le changement ne peut être subi. Il s'étudie d'ailleurs à travers trois éléments majeurs: les contingences externes, les institutions et la rationalité des acteurs. Les organisations se structurent par des compromis entre les différentes rationalités des acteurs. Ils doivent donc être capables d'interprétation et d'adaptation, car le changement ne se réalisera qu'à partir du moment où un consensus est établi. Il ne peut être mené sous la seule contrainte. Il faudra, en effet, qu'à un moment du processus, les acteurs redonnent du sens à leur action.

4 Notre travail personnel s'est enrichi de cet exposé, mais nous ne pouvons pas nous empêcher de nous interroger sur la capacité de négociation des acteurs dans des contextes de crise. Il nous apparaît en effet que certains déterminants (pour reprendre le terme de Bernoux) en limitent la portée, voire confisquent les espaces de débat. Le sens peut-il toujours être co-construit? Dans un contexte de rupture, n'est-il pas par moment imposé par le contexte ou par certains acteurs dominants? 